\title{
Donor-acceptor pair luminescence in compensated Si for solar cells
}

\author{
Michio Tajima, ${ }^{1, a)}$ Takaaki Iwai, ${ }^{1}$ Hiroyuki Toyota, ${ }^{1}$ Simona Binetti, ${ }^{2}$ and Daniel Macdonald ${ }^{3}$ \\ ${ }^{1}$ Institute of Space and Astronautical Science/JAXA, Sagamihara 252-5210, Japan \\ ${ }^{2}$ Milano-Bicocca Solar Energy Research Center (MIB-SOLAR), Department of Material Science, University \\ of Milano-Bicocca, via Cozzi 53, 20125 Milano, Italy \\ ${ }^{3}$ School of Engineering, The Australian National University, Canberra, ACT 0200, Australia
}

(Received 9 June 2011; accepted 11 July 2011; published online 17 August 2011)

\begin{abstract}
A broad band with a fine structure on the higher energy side has been commonly observed in photoluminescence at $4.2 \mathrm{~K}$ from compensated Si for solar cells involving P donors and B acceptors on the order of $10^{16} \mathrm{~cm}^{-3}$. We calculated the theoretical spectrum of donor-acceptor (DA) pair luminescence from the density distribution of pairs as a function of the transition energy of respective pairs with separations ranging from 1.9 to $3.3 \mathrm{~nm}$. A close agreement was obtained between the observed spectral structure and the theoretical curve using the generally accepted $\mathrm{P}$ donor and $\mathrm{B}$ acceptor ionization energies, where a systematic deviation was explained by the Van der Waals interaction between shallow $\mathrm{P}$ donors and $\mathrm{B}$ acceptors. This allows us to conclude that the band with the fine structure is due to the $\mathrm{P}$-donor-B-acceptor pair recombination. This identification was confirmed by the observation of As-donor-B-acceptor pair luminescence in an As-doped sample. The present findings indicate that $\mathrm{P}$ and $\mathrm{B}$ impurities with concentrations on the order of $10^{16} \mathrm{~cm}^{-3}$ are unlikely to form complexes and that their ionization energies are not changed from those in the low concentration range. (C) 2011 American Institute of Physics. [doi:10.1063/1.3622560]
\end{abstract}

\section{INTRODUCTION}

Donor-acceptor (DA) pair luminescence was investigated in detail first in GaP. ${ }^{1,2}$ Hundreds of sharp lines were observed on the higher energy side of a broad emission band, and they were unambiguously identified as being due to the radiative recombination of electrons and holes trapped at distant donors and acceptors, where the respective lines reflected the difference in the Coulombic energy in various DA pair separations. DA pair luminescence with the sharp-line structure has been found in many III-V, II-VI, and IV-IV compound semiconductors. ${ }^{3}$ However, little work has been performed on the DA pair luminescence from $\mathrm{Si}$. The main reason for this is that $\mathrm{Si}$ crystals for electronic devices have very low residual impurities, resulting in low compensation ratio. To observe the DA pair luminescence, impurity levels on the order of $10^{15}-10^{17} \mathrm{~cm}^{-3}$ are necessary for both donor and acceptor impurities, as is the case for GaP and other semiconductors. Enck and Honig first reported broadband DA pair luminescence in $\mathrm{Si}$ which was compensated by intentionally doping of various donor and acceptor impurities. ${ }^{4}$ Ziemelis et al. observed the fine structure due to DA pair luminescence in Si intentionally doped with In, Ga, or Al acceptors. ${ }^{5-7}$ The ionization energies of these acceptors are larger than the energy of the most conventional B acceptor. The involvement of deep donors and/or acceptors is highly favorable to observe discrete $\mathrm{DA}$ pair lines in $\mathrm{Si}$, as discussed later. Although $\mathrm{P}$ and $\mathrm{B}$ are the most frequently used shallow donors and acceptors in electronic industries, their DA pair luminescence had not been reported. Recently the present authors have observed a fine structure in compensated Si for

${ }^{\text {a)} E l e c t r o n i c ~ m a i l: ~ t a j i m a @ i s a s . j a x a . j p . ~}$ solar cells and identified its origin as being due to the DA pair emission involving shallow $\mathrm{P}$ donors and $\mathrm{B}$ acceptors. ${ }^{8}$

The use of low-purity grade Si materials, called "solargrade Si (SoG-Si)," is essential for the mass production of solar cells to cope with current energy and environmental issues. The concentrations of the residual donor and acceptor impurities in the SoG-Si are on the order of $10^{15}-10^{17}$ $\mathrm{cm}^{-3}$, leading to a heavy compensation between donors and acceptors. Although the impurity analysis in this concentration range is quite important, conventional impurity characterization techniques do not work satisfactorily. Resistivity measurement is ineffective for compensated samples. Secondary ion mass spectroscopy (SIMS), glow discharge mass spectroscopy (GD-MS) and inductively coupled plasma mass spectroscopy (ICP-MS) become erroneous as the concentration is decreased to the order of $10^{15} \mathrm{~cm}^{-3}$. The photoluminescence $(\mathrm{PL})^{9,10}$ and infrared absorption (IR) ${ }^{11,12}$ methods for quantitative impurity analysis are not applicable to a concentration range higher than $10^{15} \mathrm{~cm}^{-3}$. We also have to consider the formation of impurity complexes in the high concentration range. They are electrically inactive in general. Mass spectroscopies detect the total amount of impurities including those in complexes, while the PL and IR methods detect only the electrically active donor and acceptor impurities. Therefore, the successful observation of the DA pair luminescence involving $\mathrm{P}$ donors and $\mathrm{B}$ acceptors on the order of $10^{15}-10^{17} \mathrm{~cm}^{-3}$ is quite useful for developing an analytical technique for these impurities.

The purpose of this paper was to make a detailed investigation of the DA pair luminescence with the aim of accurate characterization of donor and acceptor impurities in compensated Si for solar cells, which is crucial in the fabrication of high-quality and low-cost solar cells. The paper is 
organized as follows: A brief overview is given in Sec. II of the theory of DA pair luminescence in Si. Section III describes samples and experimental apparatuses and conditions. In Sec. IV, we demonstrate a universal observation of the DA pair luminescence in SoG-Si, point out the significance of the Van der Waals interaction in shallow DA pairs, and confirm the DA pair identification. Based on these results we discuss the electrical activity of $\mathrm{P}$ and $\mathrm{B}$ impurities on the order of $10^{16} \mathrm{~cm}^{-3}$.

\section{THEORETICAL BACKGROUND}

The radiative recombination energy of an electron and a hole on an isolated distant DA pair separated by a distance $r$ is given by

$$
h v=E_{G}-\left(E_{D}+E_{A}\right)+\frac{e^{2}}{4 \pi \varepsilon_{0} \varepsilon_{r} r}-\frac{\varepsilon^{2}}{4 \pi \varepsilon_{0} \varepsilon_{r} r}\left(\frac{\alpha}{r}\right)^{5},
$$

where $E_{G}$ is the energy gap, $E_{D}$ and $E_{A}$ are the donor and acceptor ionization energy, and $\varepsilon_{r}$ is the low-frequency relative dielectric constant. ${ }^{1,2}$ The last term in Eq. (1) is the Van der Waals $(\mathrm{VdW})$ interaction and $\alpha$ is the effective VdW coefficient for a given DA pair. The theoretical spectrum of DA pair luminescence is deduced as the statistical probability of DA pairings as a function of the transition energy given by Eq. (1). Si has a diamond structure consisting of two interpenetrating face-centered cubic (fcc) lattices, offset from one another by $(1 / 4,1 / 4,1 / 4)$, where we use the ordinate unit of a lattice constant. DA pairs within the same fcc lattice denote type I pairing, while those within the different fcc lattice type II pairing. The pair separation $r$ for the two types of DA pairs is given by

$$
r=\left(\frac{1}{2} m\right)^{\frac{1}{2}} a_{0} \quad \text { (type I) }
$$

and

$$
r=\left(\frac{1}{2} m-\frac{5}{16}\right)^{\frac{1}{2}} a_{0} \quad \text { (type II), }
$$

where $m$ is a shell number for indexing the pair, the nearest neighbor pair having $m=1$, the second nearest pair $m=2$, and so on, and $a_{0}$ is a lattice constant. ${ }^{6}$ Number of pairs is calculated as a function of $m$ for type I and type II. The DA pair is classified into type I and type II categories in compound semiconductors, depending on the substitutional sites of impurities. However, such classification is meaningless in the diamond structure, since the basis of the lattice consists of identical atoms. Therefore, the theoretical spectrum of DA pair luminescence in $\mathrm{Si}$ is the sum of the type I and type II spectra. An example of the theoretical spectrum will be shown in Fig. 3(c), where the unprimed and primed numbers above several lines indicate $m$ for type I and type II, respectively.

\section{EXPERIMENTAL TECHNIQUE}

The samples used for the present study were Si wafers from ingots grown by the Czochralski method with $\mathrm{B}$ and $\mathrm{P}$
TABLE I. Donor and acceptor concentrations of $\mathrm{Si}$ crystals grown by Czochralski method. Samples no. 1021, no. 621 , no. 21 , no. 45 , and no. 44 are compensated, while sample no. 72 is uncompensated. Unimib and ANU

\begin{tabular}{|c|c|c|c|c|c|c|}
\hline \multirow[b]{2}{*}{ Sample } & \multicolumn{2}{|c|}{$N_{A}\left(10^{16} \mathrm{~cm}^{-3}\right)$} & \multicolumn{2}{|c|}{$N_{D}\left(10^{16} \mathrm{~cm}^{-3}\right)$} & \multicolumn{2}{|c|}{ Resistivity $(\Omega \mathrm{cm})$} \\
\hline & Unimib & ANU & Unimib & ANU & Unimib & ANU \\
\hline No. 1021 & 2.0 & - & 1.8 & - & - & - \\
\hline No. 621 & 1.3 & - & 0.61 & - & - & - \\
\hline No. 21 & 1.1 & - & 0.34 & - & - & - \\
\hline No. 45 & 3.0 & 4.0 & 2.2 & 2.9 & 2.01 & 1.6 \\
\hline No. 44 & 4.8 & 8.1 & 1.2 & 4.0 & 0.55 & 0.47 \\
\hline No. 72 & 1.3 & 1.7 & ND & ND & 1.12 & 1.0 \\
\hline
\end{tabular}
data were obtained by lifetime (Ref. 13) and Hall effect (Ref. 14) measurements, respectively.

doping. The $\mathrm{B}$ and $\mathrm{P}$ concentrations were determined to be on the order of $10^{15}-10^{17} \mathrm{~cm}^{-3}$ from the lifetime ${ }^{13}$ and Hall effect ${ }^{14}$ measurements, as shown in Table I. We also measured various compensated $\mathrm{Si}$ for solar cells grown by the unidirectional solidification method. Their dominant impurities were $\mathrm{P}$ and $\mathrm{B}$ on the order of $10^{15}-10^{17} \mathrm{~cm}^{-3}$ from the SIMS and ICP-MS measurements. Essentially the same spectra with the fine structure were observed from most of the samples. One of the samples (no. J03) was unintentionally doped with an exceptionally high concentration of As, which was useful to investigate the spectral shift originating from the difference in ionization energy between $\mathrm{P}$ and As donors. The $\mathrm{B}, \mathrm{P}$, and As concentrations in this sample were determined to be $1.6,2.1$, and $7.2 \times 10^{15} \mathrm{~cm}^{-3}$, respectively, from the SIMS measurement.

Most of the PL measurements were performed at $4.2 \mathrm{~K}$, while the samples were immersed in liquid $\mathrm{He}$ in a quartz cryostat. For measurements at higher temperatures, the samples were mounted on a He closed-cycle type of cryostat. We excited them with the $532 \mathrm{~nm}$ line of second harmonic generation of a $\mathrm{Nd}: \mathrm{YVO}_{4}$ laser with a beam diameter of about $3 \mathrm{~mm}$. The incident power on the sample surface was 35 and $0.7 \mathrm{~mW}$ for high and low excitation conditions, respectively. Emission from the sample was analyzed with a monochromator $(f=0.32 \mathrm{~m})$ with 300 and 600 grooves $\mathrm{mm}^{-1}$ gratings blazed at $1.2 \mu \mathrm{m}$ and detected by a cooled InGaAs photodiode array. The spectral resolution was about 0.25 and $0.125 \mathrm{meV}$, depending on the grating used.

\section{RESULTS AND DISCUSSION}

PL spectra from the samples under the spectral resolution of $0.25 \mathrm{meV}$ are shown in Fig. 1, where the blue and red curves (the black and gray curves in the printed version) correspond to the high and low excitation conditions. Sample no. 72 was B-doped and uncompensated, and hence sharp Bbound exciton lines appeared: $\mathrm{B}_{\mathrm{NP}}(\mathrm{BE}), \mathrm{B}_{\mathrm{TA}}(\mathrm{BE})$, and $\mathrm{B}_{\mathrm{TO}}(\mathrm{BE})$ are the no-phonon (NP) band and its transverse acoustic (TA) and the transverse optical (TO) phonon sidebands, respectively. ${ }^{15} \mathrm{~A}$ series of sharp lines following the bound exciton lines are due to the bound multiple exciton complexes (BMEC). In the other compensated samples, three bands appeared at 1.098, 1.079, and $1.041 \mathrm{eV}$ under the low excitation condition with a similar shape having a tail on the 
high-energy side. The spacing between the highest and middle bands and that between the highest and lowest bands is in good agreement with the TA and TO phonon energies (19 and $58 \mathrm{meV}$ ), respectively. This allows us to conclude that the highest, middle and lowest bands are a NP band and its TA and TO phonon sidebands, respectively. The peak positions of the three bands shifted equally to the high-energy side under the high excitation condition; this is characteristic of DA pair luminescence. ${ }^{2,3}$ The peak positions of the three bands agree with the $\mathrm{P}$-donor and $\mathrm{B}$-acceptor pair $(\mathrm{P}-\mathrm{B}$ pair) luminescence reported by Enck and Honig. ${ }^{4}$ Based on these facts we conclude that the observed bands are due to $\mathrm{P}-\mathrm{B}$ pair luminescence; we hereafter label the three bands as $\mathrm{P}-\mathrm{B}_{\mathrm{NP}}, \mathrm{P}-\mathrm{B}_{\mathrm{TA}}$, and $\mathrm{P}-\mathrm{B}_{\mathrm{TO}}$.

In addition to the $\mathrm{P}-\mathrm{B}$ bands we could observe a sharp line at $1.1500 \mathrm{eV}$ and three broad bands at 1.145, $1.087 \mathrm{eV}$ and vaguely at $1.125 \mathrm{eV}$ under the high excitation condition in Fig. 1. They are assigned as the NP line of P-bound exciton, denoted $\mathrm{P}_{\mathrm{NP}}(\mathrm{BE})$ and the NP, TO, and TA bands of the luminescence due to impurity associated localized carriers (IALC), ${ }^{16}$ respectively, from their peak positions. A systematic spectral shift to the lower energy side and broadening were observed for the IALC bands with increase in the impurity concentration, as reported preliminarily elsewhere. ${ }^{17}$ These bands will not be discussed further, since this is outside the scope of the present paper.

Now we focus on the fine structure in the high-energy tail of the NP and TO bands of the $\mathrm{P}-\mathrm{B}$ pair luminescence.

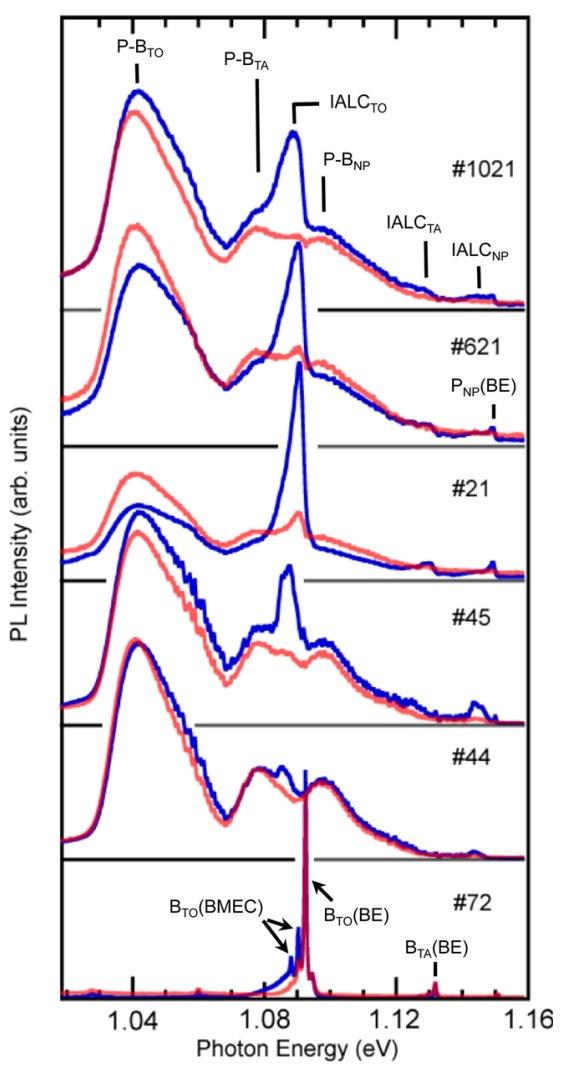

FIG. 1. (Color online) PL spectra of compensated Si involving P donors and B acceptors (samples no. 1021, no. 621, no. 21 , no. 45 , and no. 44) and uncompensated B-doped Si (sample no. 72) at $4.2 \mathrm{~K}$ under high and low excitation conditions (blue and red curves in online version and black and gray curves in printed version, respectively). Samples are summarized in Table I.
The shape of the structure of the NP band was identical with that of the TO bands. ${ }^{8}$ The structure appeared under both high and low excitation conditions in the NP and TO bands, as shown in Fig. 1. Exactly the same structure was observed on the higher energy side of the TO band in various compensated samples, as shown in Fig. 2. Sample no. 45 is the one investigated in detail in the following part.

Figure 3(a) is an enlarged illustration of the high-energy side of the NP band in sample no. 45. Many narrow discrete bands are observable. The theoretical discrete line structure of the DA pair luminescence is shown in Fig. 3(c), where the unprimed and primed numbers above several lines indicate $m$ for type I and type II, respectively. In the figure we used the values of $\varepsilon_{r}=11.7$ and $a_{0}=0.543 \mathrm{~nm}$, neglected the VdW term, and adjusted the $\left[E_{G}-\left(E_{D}+E_{A}\right)\right]$ value in Eq. (1), which will be described later. The distribution of pairs is very dense. This is due to the mixture of type I and type II spectra, and to the small value of $\left(E_{D}+E_{A}\right)$ as a result of shallow levels, where only the distant pairs $(m \geq 20)$ contribute to the emission spectrum.

The observed widths of discrete narrow bands in Fig. 3(a) were on the order of $0.25-0.5 \mathrm{meV}$. We measured the spectrum under the twice-higher spectral resolution of $0.125 \mathrm{meV}$, but did not observe any substantial spectral narrowing, indicating that the observed curve reflected the real spectral shape at $4.2 \mathrm{~K}$. Therefore, we could not make a oneto-one correspondence between the theoretical lines and the observed discrete lines as in the normal analytical procedure for the DA pair luminescence. Ziemelis and Parsons have successfully observed and identified the discrete lines for $\mathrm{P}$-In pairs with a separation ranging from 0.77 to $2.0 \mathrm{~nm}$ $(4 \leq m \leq 26){ }^{5}$ However, they could not resolve discrete lines with the separation larger than $2.0 \mathrm{~nm}$; this was the region

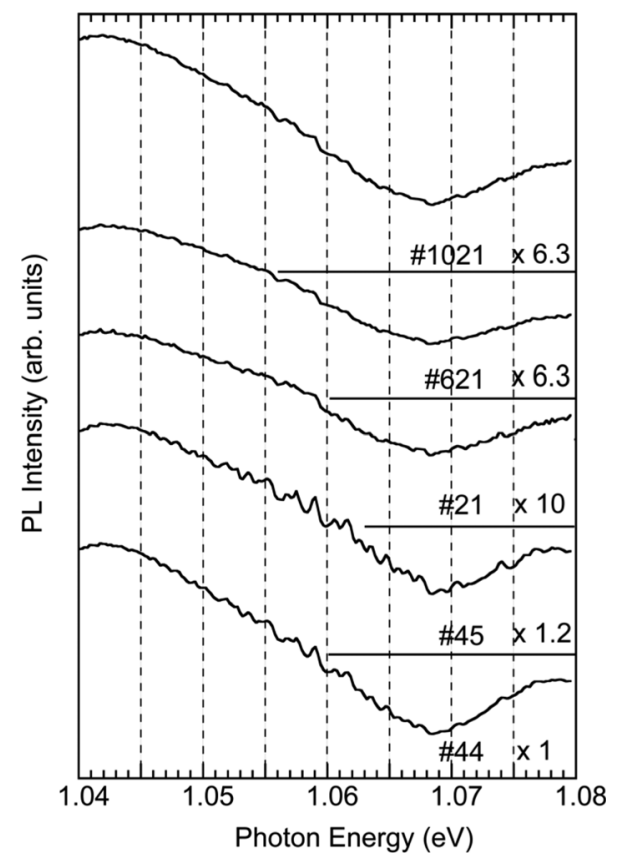

FIG. 2. Higher energy side of TO-phonon sideband of DA pair luminescence spectra in various compensated Si involving $\mathrm{P}$ donors and $\mathrm{B}$ acceptors at $4.2 \mathrm{~K}$ under high excitation condition. Symbol " $\times 10$ " denotes relative amplitude factor. 
(c)

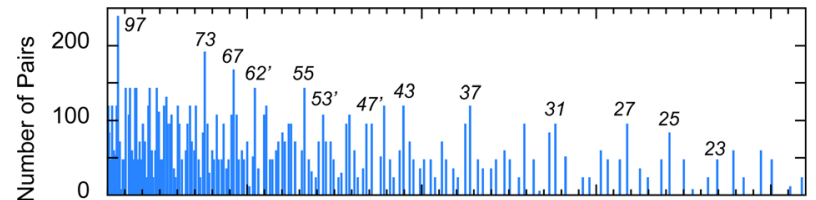

(b)

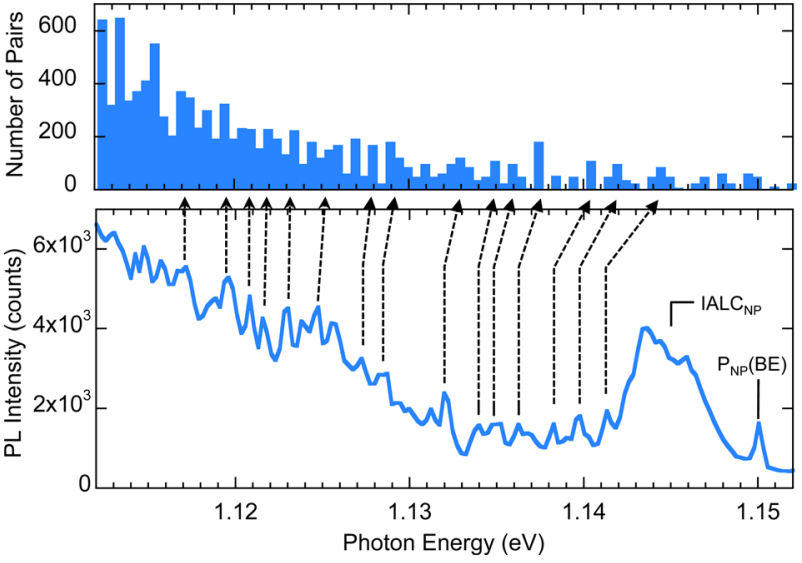

FIG. 3. (Color online) Match between the observed fine structure of DA pair luminescence and theoretical distribution of DA pairs. (a) Higher energy side of NP-band of $\mathrm{P}-\mathrm{B}$ pair luminescence spectrum at $4.2 \mathrm{~K}$ under high excitation condition. (b) Density distribution of DA pairs against photon energy calculated from the theoretical line spectrum in (c). (c) Distribution of DA pairs as a function of the transition energy of separated pairs. Unprimed and primed numbers above several lines indicate the shell number for type I and type II pairs, respectively. Theoretical spectrum in (b) was best fitted to the observed spectrum in (a) under the condition of $E_{G}-\left(E_{D}+E_{A}\right)=1.0800 \mathrm{eV}$.

that we had to analyze. In our case of P-B pairs the separation corresponding to the observed structure was estimated to range from 1.9 to $3.3 \mathrm{~nm}(25 \leq m \leq 73)$, as shown in Fig. 3 . In order to make a realistic comparison between the theoretical and observed spectra we totaled the number of pairs in each $0.5 \mathrm{meV}$ interval of photon energy, as shown in Fig. 3(b). The envelope curve of the bar graph corresponds to the pair density distribution against photon energy. Then, we were able to fit the theoretical graph to the observed spectrum by using the $\left[E_{G}-\left(E_{D}+E_{A}\right)\right]$ value as an adjustable parameter. Actually, the spectrum in Fig. 3(b) is the best-fitted one under the condition of

$$
E_{G}-\left(E_{D}+E_{A}\right)=1.0800(\mathrm{eV}) .
$$

The fine structure in the observed spectrum coincides with the theoretical curve as indicated by arrows, where the systematic deviation arises from the VdW term in Eq. (1): the observed spectrum shifted to the lower energy side and the amount of the shift became larger for smaller $m$.

Analysis of the systematic shift enables us to determine $\alpha$, the effective $\mathrm{VdW}$ coefficient for $\mathrm{P}-\mathrm{B}$ pairs. Figure 4 shows a comparison between the observation and the theoretical prediction of the recombination energy of the pairs with various separations. Energies of respective peaks were plotted against the separation calculated from Eqs. (2) and (3), where the $m$ values were determined from the spectral fit among Figs. 3(a)-3(c). Primed and unprimed numbers of $m$ denote the pairs of type I and type II, respectively. A dotted line was obtained from Eq. (1) without the VdW term, where a systematic deviation was observed as in the comparison in

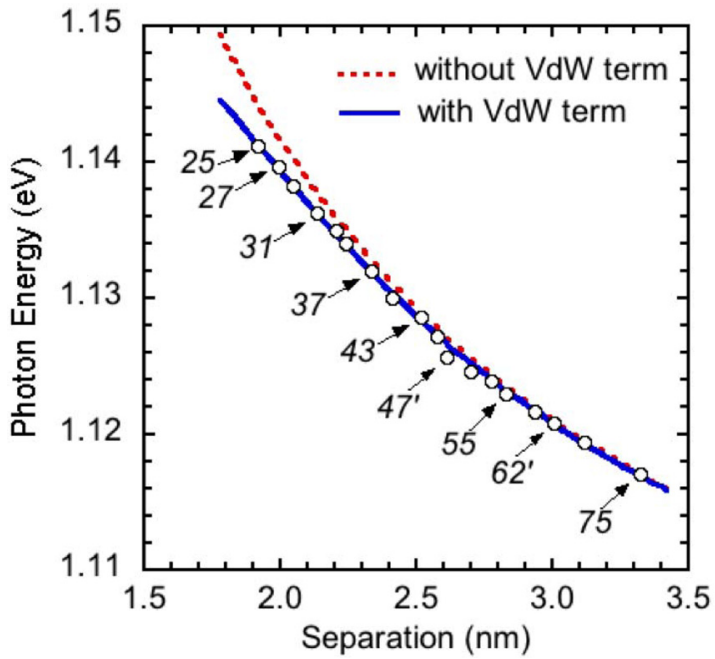

FIG. 4. (Color online) Respective peak energies of the fine structure as a function of the DA pair separation based on the peak assignment shown in Fig. 3. Solid and dotted curves represent the theoretical fit of Eq. (1) with and without the VdW term. The effective VdW coefficient $\alpha$ was determined to be $1.04 \pm 0.01 \mathrm{~nm}$ from the best fit.

Figs. 3(b) and 3(c). We included the VdW term and fitted it to the observed energies using $\alpha$ as an adjustable parameter. A very precise fit was obtained for $\alpha=1.04 \pm 0.01 \mathrm{~nm}$, as shown by a solid line in Fig. 4. This is in contrast with the results reported by Ziemelis et al. ${ }^{7}$ The effect of the VdW interaction was very small for DA pairs involving deep In, $\mathrm{Ga}$, and $\mathrm{Al}$ acceptors. We believe this is due to the difference in the polarization interaction in DA pairs: large for DA pairs consisting of shallow $\mathrm{P}$ donors and $\mathrm{B}$ acceptors in our case and small for those involving deep acceptors in their case. It is worthwhile pointing out that $\alpha=1.11 \mathrm{~nm}$ for shallow DA pairs in $\mathrm{GaP}^{2}$

To confirm the identification of the DA pair luminescence we measured a PL spectrum of an As-doped sample (no. J03) and compared it with that of sample no. 45. The PL from sample no. J03 was dominated by the BE lines and no DA pair bands were observed at $4.2 \mathrm{~K}$. This occurred as a result of the insufficient concentration of $\mathrm{B}$ acceptors in the sample. We raised the sample temperature to $10 \mathrm{~K}$ so that the $\mathrm{BE}$ lines were decreased and the DA pair bands gained in intensity. A balance between the BE and DA pair luminescence was discussed by Ziemelis and Parsons ${ }^{5}$ and will be described in more detail in a forthcoming paper as it relates to the development of the analytical technique for a high concentration of impurities in compensated Si. The TO-phonon sideband of the DA pair luminescence from sample no. J03 at $10 \mathrm{~K}$ is shown in Fig. 5. We also replotted the $\mathrm{P}-\mathrm{B}_{\mathrm{TO}}$ band of sample no. 45 at $4.2 \mathrm{~K}$ from Fig. 1. Both spectra were taken under the high excitation condition. Most of the peaks in the characteristic fine structure on the higher energy side of the DA pair luminescence coincided with each other, as shown by arrows in Fig. 5, if the $\mathrm{P}-\mathrm{B}_{\mathrm{TO}}$ band was shifted to the lower energy side by $7 \mathrm{meV}$. This shift value is nearly equal to the difference of the ionization energies between the $\mathrm{P}$ and As donors $(8 \mathrm{meV})$. It should be noted that the temperature dependence of the bandgap is negligible at temperatures lower than $20 \mathrm{~K}$. This systematic shift allowed us to confirm that the emission bands 


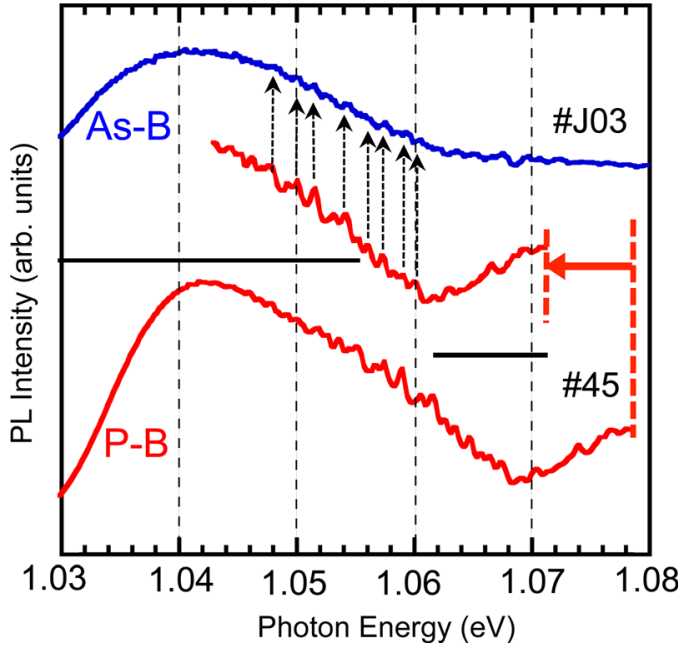

FIG. 5. (Color online) DA pair spectrum at $10 \mathrm{~K}$ from sample no. J03 doped with As, $\mathrm{P}$, and B and that at $4.2 \mathrm{~K}$ from sample no. 45 doped with $\mathrm{P}$ and $\mathrm{B}$. A part of the latter spectrum has been shifted to the lower energy side by 7 $\mathrm{meV}$ for comparison.

discussed in the present paper are DA pair luminescence due to $\mathrm{P}-\mathrm{B}$ and $\mathrm{As}-\mathrm{B}$ pairs. The fine structure due to the $\mathrm{P}-\mathrm{B}$ pair luminescence was superimposed on the As-B pair luminescence in sample no. J03, for example at around $1.068 \mathrm{eV}$ in Fig. 5, which was due to the presence of $\mathrm{P}$ donors in addition to As donors in the sample.

We recognized a slight discrepancy between the theoretical and observed spectra. The most likely source of the discrepancy is the disregard of the dependence of the transition probability on the DA pair separation, which induces the differences in electron and hole capture rates. Other possible sources are the difference in density between the possible DA pair sites and the actual DA pair distribution, and the higher order complexity, such as inequivalent sites in a given $m$ shell and the transition from excited states. The condition in Eq. (4) is consistent with the generally accepted values of $\mathrm{P}$ donor, $\mathrm{B}$ acceptor ionization energies ${ }^{18}$ and bandgap energy $^{19}\left(E_{D}=45 \mathrm{meV}, E_{A}=45 \mathrm{meV}\right.$, and $E_{G}(0 \mathrm{~K})$ $=1.1700 \mathrm{eV}$ ).

The present findings give us insight into the electronic properties of donor and acceptor impurities in the high concentration range. The estimated $\left(E_{D}+E_{A}\right)$ value for $\mathrm{P}$ donors and $\mathrm{B}$ acceptors in the present samples with the concentrations on the order of $10^{16} \mathrm{~cm}^{-3}$ is consistent with the $E_{D}$ and $E_{A}$ values in the low concentration range $\left(\leq 10^{15} \mathrm{~cm}^{-3}\right)$. This indicates that the reduction of the ionization energy due to high doping does not occur in the present concentration range. This hypothesis is supported by the simulation by Altermatt $e t a l .^{20}$ : such a reduction occurs at the concentration range higher than $10^{17} \mathrm{~cm}^{-3}$. The observation of discrete DA pair lines in the present samples indicate that both $\mathrm{P}$ and $\mathrm{B}$ impurities act as isolated donors and acceptors, respectively, but do not make an atomic complex which presumably loses the original electrical activities of $\mathrm{P}$ donors and $\mathrm{B}$ acceptors. We observed a systematic variation among the BE lines, the IALC band and the DA pair bands depending on the impurity concentrations and their compensation ratios, which will be reported in a forthcoming paper. The systematic change could be expected from the monotonic increases in the donor and acceptor concentrations but not from the complex formation.

\section{CONCLUSIONS}

We have analyzed the fine structure observed commonly in the higher energy side of the $\mathrm{P}-\mathrm{B}$ pair luminescence at 4.2 $\mathrm{K}$ in compensated $\mathrm{Si}$ for solar cells involving $\mathrm{P}$ donors and $\mathrm{B}$ acceptors on the order of $10^{16} \mathrm{~cm}^{-3}$. A close agreement was obtained between the observed structure and the theoretical spectrum deduced from the density distribution of discrete DA pairs as a function of the transition energy of respective pairs with separations ranging from 1.9 to $3.3 \mathrm{~nm}$. Substantial contribution of the VdW interaction was recognized with effective VdW coefficient of $1.04 \pm 0.01 \mathrm{~nm}$ for $\mathrm{P}-\mathrm{B}$ pairs. The present identification was confirmed by the systematic spectral shift observed for the As-B pair luminescence. A clear observation of the fine structure due to the discrete DA pair luminescence in compensated $\mathrm{Si}$ is strong evidence for the idea that both $\mathrm{P}$ and $\mathrm{B}$ impurities act as isolated donors and acceptors, respectively, with the same ionization energies as in the low concentration range, but do not form complexes.

\section{ACKNOWLEDGMENTS}

This work was partly supported by the New Energy and Industrial Technology Development Organization (NEDO) under the Ministry of Economy, Trade and Industry (METI).

${ }^{1}$ J. J. Hopfield, D. G. Thomas, and M. Gershenzon, Phys. Rev. Lett. 10, 162 (1963).

${ }^{2}$ D. G. Thomas, M. Gershenzon, and F. A. Trumbore, Phys. Rev. 133, A269 (1964).

${ }^{3}$ P. J. Dean, Progress in Solid State Chemistry, (Elsevier, New York, 1973), Vol. 8, p. 1.

${ }^{4}$ R. C. Enck and A. Honig, Phys. Rev. 177, 1182 (1969).

${ }^{5}$ U. O. Ziemelis and R. R. Parsons, Can. J. Phys. 59, 784 (1981).

${ }^{6}$ U. O. Ziemelis, M. L. W. Thewalt, and R. R. Parsons, Appl. Phys. Lett. 39, 972 (1981).

${ }^{7}$ U. O. Ziemelis, M. L. W. Thewalt, and R. R. Parsons, Can. J. Phys. 60, 1041 (1982).

${ }^{8}$ M. Tajima, T. Iwai, H. Toyota, S. Binetti, and D. Macdonald, Appl. Phys. Express 3, 071301 (2010).

${ }^{9}$ M. Tajima, Appl. Phys. Lett. 32, 719 (1978).

${ }^{10}$ Semiconductor Equipment and Materials International, SEMI MF13890704 (2004).

${ }^{11}$ B. O. Kolbesen, Appl. Phys. Lett. 27, 353 (1975).

${ }^{12}$ Semiconductor Equipment and Materials International, SEMI MF16301107 (2004).

${ }^{13}$ D. Macdonald, A. Cuevas, and L. J. Geerligs, Appl. Phys. Lett. 92, 202119 (2008).

${ }^{14}$ S. Binetti, A. Le Donne, M. Acciarri, D. Macdonald, T. Iwai, and M. Tajima, presented at E-MRS 2010 Spring Meeting, Strasbourg, 2010, No. I4-2.

${ }^{15}$ K. Kosai and M. Gershenzon, Phys. Rev. B 9, 723 (1974).

${ }^{16}$ M. A. Vouk and E. C. Lightowlers, J. Lumin. 15, 357 (1977).

${ }^{17} \mathrm{~T}$. Iwai, M. Tajima, and A. Ogura, The Forum on the Science and Technology of Silicon Materials 2010, November 14-17, 2010 (Okayama University, Japan, 2010), p. 394.

${ }^{18}$ Landolt-Börnstein, New Series, edited by O. Madelung, (Springer-Verlag, Berlin, 1982), Vol. III/17a, p. 43.

${ }^{19}$ W. Bludau, A. Onton, and W. Heinke, J. Appl. Phys. 45, 1846 (1974).

${ }^{20}$ P. P. Altermatt, A. Schenk, and G. Heiser, J. Appl. Phys. 100, 113714 (2006). 